\title{
ON THE LEXICOGRAPHIC DEGREE OF TWO-BRIDGE KNOTS
}

\author{
ERWAN BRUGALLÉ, PIERRE-VINCENT KOSELEFF, AND DANIEL PECKER
}

\begin{abstract}
We study the degree of polynomial representations of knots. We obtain the lexicographic degree for two-bridge torus knots and generalized twist knots. The proof uses the braid theoretical method developed by Orevkov to study real plane curves, combined with previous results from [KP10] and [BKP14]. We also give a sharp lower bound for the lexicographic degree of any knot, using real polynomial curves properties.
\end{abstract}

\section{INTRODUCTION}

It is known that every knot in $\mathbf{S}^{3}$ can be represented as the closure of the image of a polynomial embedding $\mathbb{R} \rightarrow \mathbb{R}^{3} \subset \mathbf{S}_{3}$, see [Sh92, Va90]. In these early papers on the subject, only a few specific examples were given. The two-bridge torus knots form the first infinite family of knots for which a polynomial representation was explicitly given, see [RS96, KP08, KP11, KP10].

Example 1.1 (Harmonic knots $([\mathrm{KP} 11, \mathrm{KP} 12]))$. The harmonic knot $\mathrm{H}(a, b, c)$ is the polynomial knot parametrized by $\left(T_{a}(t), T_{b}(t), T_{c}(t)\right)$, where $T_{n}$ is the classical Chebyshev polynomial $T_{n}(\cos t)=\cos n t$ and $a, b, c$ are pairwise coprime integers. The two knot diagrams depicted in Figure 1 are the $x y$-diagrams of the knots

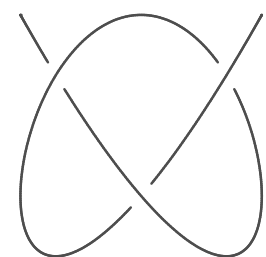

$3_{1}=\mathrm{H}(3,4,5)$

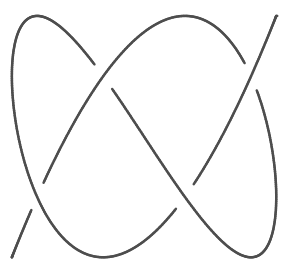

$4_{1}=\mathrm{H}(3,5,7)$

Figure 1. Two harmonic knots

$\mathrm{H}(3,4,5)$ and $\mathrm{H}(3,5,7)$, corresponding to the projection $(x, y, z) \mapsto(x, y)$.

The multidegree of a polynomial map $\gamma: \mathbb{R} \rightarrow \mathbb{R}^{n}, t \mapsto\left(P_{i}(t)\right)$ is the $n$-tuple $\left(\operatorname{deg}\left(P_{i}\right)\right)$. The lexicographic degree of a knot $K$ is the minimal multidegree, for the lexicographic order, of a polynomial knot whose closure in $\mathbf{S}^{3}$ is isotopic to $K$. The unknot has lexicographic degree $(-\infty,-\infty, 1)$, and one sees easily that the lexicographic degree of any other knot is $(a, b, c)$ with $3 \leq a<b<c$. Given a knot, it is in general a difficult problem to determine its lexicographic degree.

Date: July 13, 2017

2000 Mathematics Subject Classification. 14H50, 57M25, 11A55, 14P99.

Key words and phrases. Real pseudoholomorphic curves, polynomial knots, two-bridge knots. 
In particular, the corresponding diagram might not have the minimal number of crossings.

In this paper we investigate the case of two-bridge knots, that are the knots for which $a=3$, see [Cr04, KP10, BKP14]. We focus in particular on two-bridge torus knots and generalized twist knots, that we respectively denote by $C(m)$ with $m$ odd, and $C(m, n)$ with $m n$ positive and even (see Figure 2). Note that $C(-m)$ and $C(-m,-n)$ are the mirror images of $C(m)$ and $C(m, n)$.
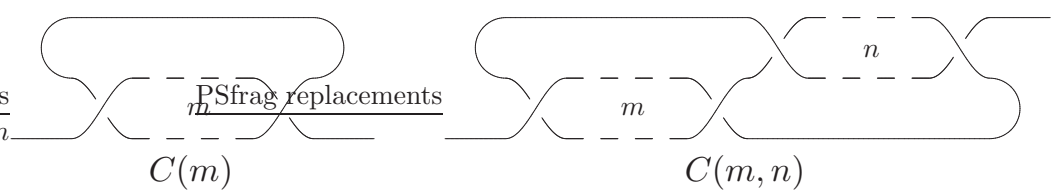

Figure 2. Two-bridge torus knots and generalized twist knots

Figure 3 shows typical examples, $C(5)$ and $C(4,3)$.

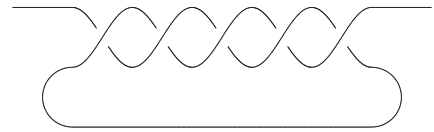

$5_{1}=C(5)$

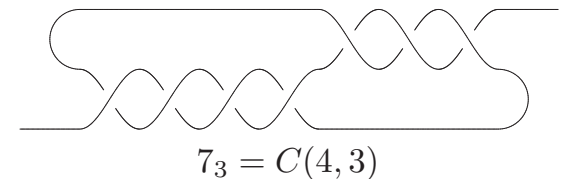

$7_{3}=C(4,3)$

Figure 3 . The knots $5_{1}$ and $7_{3}$

Mishra claimed in [Mi00] that the lexicographic degree of the torus knot $C(m)$, $m \not \equiv 2(\bmod 3)$, is $(3, m+1, c)$. Later on, it was proved in [KP08] that this is false for every integer $m>5$. At that stage, the lexicographic degree of only a few knots was known, namely the trefoil, the figure-eight knot, the torus knot $5_{1}$ [Mi00], and the torus knot $7_{1}[\mathrm{KP} 08]$.

For every two-bridge knot of crossing number $N$, a polynomial representation of degree $(3, b, c)$, with $b+c=3 N$ and $b \geq N+1$ is constructed in [KP10]. From that construction an upper bound for the lexicographic degree of two-bridge knots is immediately deduced. The main result of this paper is that this upper bound is sharp for two-bridge torus knots and for generalized twist knots.

Theorem 3.9. Let $K$ be the two-bridge knot $C(m)$, or $C(m, n)$ with $m n>0$, and let $N$ be its crossing number. Then the lexicographic degree of $K$ is

$$
\left(3,\left[\frac{3 N-1}{2}\right],\left[\frac{3 N}{2}\right]+1\right) .
$$

The proof goes by the study of the plane curve defined by the $x y$-projection of a polynomial knot, and uses Orevkov's braid theoretical approach to study real plane curves (see [Ore99] or Section 2). Like in the case of knots, we define the lexicographic degree of a long knot diagram as the minimal multidegree, for the lexicographic order, of a polynomial knot whose $x y$-diagram is isotopic in $\mathbb{R}^{2}$ to $D$. 
The main idea in the proof of Theorem 3.9 is as follows. Suppose that $D_{1}$ is a trigonal long knot diagram with lexicographic degree $(3, b, c)$. If $D_{2}$ is a trigonal diagram obtained from $D_{1}$ by an isotopy through trigonal diagrams which never increases the number of crossings, then one may expect that it has lexicographic degree $\left(3, b^{\prime}, c^{\prime}\right)$ with $b^{\prime} \leq b$. If this was the case, then Theorem 3.9 would follow from the study of the alternating diagrams $C(m)$ or $C(m, n)$, combined with the following theorem.

Theorem A. ([BKP14]) Let $K$ be the two-bridge knot $C(m)$, or $C(m, n)$ with $m n>0$, and let $D$ be a trigonal diagram of $K$. Then it is possible to transform $D$ into an alternating trigonal diagram, so that all intermediate diagrams remain trigonal, and the number of crossings never increases.

Unfortunately, one cannot ensure that the lexicographic degree of a trigonal knot diagram does not increase during an isotopy, even if this isotopy never increases the number of crossings. For this to be true, one needs to enlarge the set of objects we are studying, namely we have to consider real pseudoholomorphic curves (see Corollary 3.7). Note that a real algebraic curve is real pseudoholomorphic, however the converse is not true in general.

We conclude the paper by providing a lower bound for the lexicographic degree of every non-trivial knot. We also give the lexicographic degree of some infinite families of knots, showing in particular that this lower bound is sharp.

Theorem 4.3. Let $K$ be a knot of crossing number $N>0$. Then the lexicographic degree of $K$ is at least $(3, N+1,2 N-1)$. For every integer $N \not \equiv 2(\bmod 3), N \geq 3$, there exists a knot of crossing number $N$ and lexicographic degree $(3, N+1,2 N-1)$.

The paper is organized as follows. We recall Orevkov's braid theoretical method in Section 2. To avoid technical difficulties coming from an excess of generality, we restrict ourselves to the case of real curves of bidegree $(3, b)$, whereas the whole section generalizes to all bidegrees. We prove Theorem 3.9 in Section 3. To keep the exposition as light as possible we keep working with real algebraic curves as long as pseudoholomorphic curves are not needed. In particular we prove the lower bound of Theorem 3.9 in the particular case of the diagrams $C(m)$ and $C(m, n)$ for real algebraic curves (Proposition 3.1). We introduce real pseudoholomorphic curves only in Section 3.2 to deduce the general lower bound of Theorem 3.9 from Proposition 3.1 (Proposition 3.8). In Section 4, we first obtain a sharp lower bound for alternating diagrams. The proof is elementary, it is based on polynomial plane curves properties. As a consequence, we deduce Theorem 4.3 and an upper bound for the crossing number of alternating knots of polynomial degree $d$.

\section{The BRAid of a REAL Algebraic CuRVe in $\mathbb{C}^{2}$}

Here we recall basic facts about the braid theoretical approach developed by Orevkov to study real algebraic curves in $\mathbb{C}^{2}$.

2.1. Link associated to a real algebraic curve. Given $C \subset \mathbb{C}^{2}$ a real algebraic curve, we denote by $\mathbb{R} C$ the real part of $C$, i.e. $\mathbb{R} C=C \cap \mathbb{R}^{2}$. Let us fix an orientation preserving diffeomorphism $\Phi: \mathbb{R}^{4} \rightarrow\left\{(x, y) \in \mathbb{C}^{2} \mid \operatorname{Im}(x)>0\right\}$, and let us denote by $B_{r}$ the image by $\Phi$ of the 4 -ball of radius $r$, and by $S_{r}=\partial B_{r}$ the 
image by $\Phi$ of the 3 -sphere of radius $r$. If $C \subset \mathbb{C}^{2}$ is a real algebraic curve, then all links $S_{r} \cap C$ (resp. all surfaces $B_{r} \cap C$ ) are isotopic if $r$ is large enough.

Definition 2.1. The link $L_{C}=S_{r} \cap C$, for $r$ large enough, is called the link associated to the real algebraic curve $C$.

We denote $U_{C}=B_{r} \cap C$, and we orient $L_{C}$ as the boundary of $U_{C}$. We will use the following standard proposition in the proof of Theorem 3.9. The linking number of two oriented links $L_{1}$ and $L_{2}$ is denoted by $\mathrm{lk}\left(L_{1}, L_{2}\right)$; the algebraic intersection number of two smooth surfaces $U_{1}$ and $U_{2}$ in $B_{r}$ intersecting transversally and in $B_{r} \backslash S_{r}$ is denoted by $U_{1} \cdot U_{2}$.

Proposition 2.2. If $L_{1}$ and $L_{2}$ are two sublinks of $L_{C}$ which bound two components $U_{1}$ and $U_{2}$ of $N_{C}$, then

$$
\operatorname{lk}\left(L_{1}, L_{2}\right)=U_{1} \cdot U_{2} .
$$

It turns out that in some situations, one can read directly on the real part $\mathbb{R} C$ an expression of the oriented link $L_{C}$ as a closed braid. For the sake of simplicity, we restrict ourselves here to the very special case of trigonal rational curves. For the general case, we refer to [Ore99]. In what follows, we are interested in curves in $\mathbb{C}^{2}$ up to isotopies respecting the fibration of $\mathbb{C}^{2}$ by vertical lines.

Definition 2.3. An isotopy on a curve $C \subset \mathbb{C}^{2}$ is called a $\mathcal{L}$-isotopy if it commutes with the projection $\pi: \mathbb{C}^{2} \rightarrow \mathbb{C},(x, y) \mapsto x$.

Let us consider a polynomial map

$$
\begin{array}{rlc}
\gamma: \mathbb{C} & \longrightarrow & \mathbb{C}^{2} \\
t & \longmapsto(P(t), Q(t))
\end{array}
$$

where $P(t)$ and $Q(t)$ are two real polynomials of degrees 3 and $b \geq 1$. Replacing $P(t)$ with $-P(t)$ if necessary, we may assume that $P(t)$ is positive for $t$ large enough. We may also assume that $b=3 k-1$ or $b=3 k-2$. If $b=3 k$, then we can replace the map $\gamma$ by the map $t \rightarrow\left(P(t), Q(t)-\alpha P^{k}(t)\right)$. In the target space $\mathbb{C}^{2}$, this corresponds to performing the real change of coordinates $y=y-\alpha x^{k}$ and does not affect the topology of the real algebraic curve $C=\gamma(\mathbb{C})$ in $\mathbb{C}^{2}$.

We suppose in addition that the map $\gamma$ is generic enough so that:

$-\gamma$ is an immersion;

— if $\gamma\left(t_{1}\right)=\gamma\left(t_{2}\right)$ for $t_{1} \neq t_{2}$, then $\gamma^{\prime}\left(t_{1}\right) \neq \gamma^{\prime}\left(t_{2}\right)$ and $\gamma^{-1}\left(\gamma\left(t_{1}\right)\right)=\left\{t_{1}, t_{2}\right\}$.

In other words, the only singularities of the embedded algebraic curve $C \subset \mathbb{C}^{2}$ are nodes. Since $C$ is real, it has three kinds of nodes:

(1) the intersection of two real branches of $C$ (i.e. $t_{1}, t_{2} \in \mathbb{R}$ ); such a real node is called a crossing, see Figure 4a;

(2) the intersection of two complex conjugated branches of $C$ (i.e. $t_{2}=\overline{t_{1}}$ ); such a real node is called solitary node, see Figure $4 \mathrm{~b}$;

(3) the intersection of two branches of $C$ which are neither real nor complex conjugated; all such nodes lie in $\mathbb{C}^{2} \backslash \mathbb{R}^{2}$ and come in pairs of complex conjugated nodes.

We denote by $N$ the number of crossings of $C$, by $\alpha$ the number of solitary nodes of $C$ and by $2 \beta$ its number of nodes lying in $\mathbb{C}^{2} \backslash \mathbb{R}^{2}$. Hence the real part $\mathbb{R} C$ is the union of $\gamma(\mathbb{R})$ together with the $\alpha$ solitary nodes of $C$.

Lemma 2.4. Given a map $\gamma: \mathbb{C} \rightarrow \mathbb{C}^{2}$ as above, we have $b-1=N+\alpha+2 \beta$. 


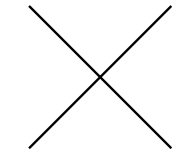

a) A crossing

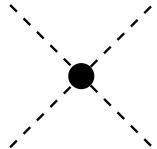

b) A solitary node

FIGURE 4.

Proof. The Newton polygon $T$ of the curve $\gamma(C)$ is the triangle with vertices $(0,0),(0,3)$ and $(b, 0)$, and the number of nodes of $\gamma(C)$ is the number of points in $\mathbb{Z}^{2}$ contained in the interior of $T$.

Example 2.5. Let $1 / \sqrt{3}<\nu<1$. Using elementary elimination theory, one sees that the real part of the parametrized curve $\gamma: t \mapsto\left(T_{3}(t), T_{4}(t+\nu)\right)$ is depicted, up to $\mathcal{L}$-isotopy, in Figure 5 a. It has two crossings and one solitary node.

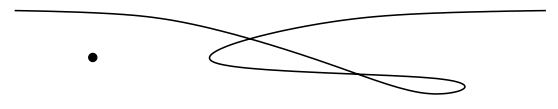

a) $\mathbb{R} C$

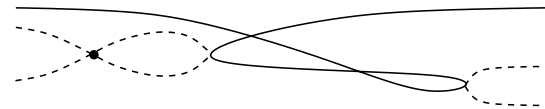

b) $A$

FiguRE 5 .

Now let us consider the set $A=\pi^{-1}(\mathbb{R}) \cap C$. It consists in the real part $\mathbb{R} C$ together with some pairs of complex conjugated arcs, meeting at critical points of the map $\pi_{\mid \mathbb{R} C}$ (i.e. at points of $\mathbb{R} C$ which intersect a vertical line non-transversely).

Example 2.6. The set $A$ in the case of the parametrization of Example 2.5 is depicted in Figure 5b. The dotted curves correspond to complex conjugate arcs.

The set $A$ is not a manifold because of critical points of the map $\pi_{\mid \mathbb{R} C}$. However there is a canonical way to perturb $A$ to a 1 -dimensional manifold and to recover the link $L_{C}$. Let $R_{1}, R_{2}$ and $\varepsilon$ be some positive real numbers, and let

$$
V_{1}=\pi^{-1}\left(\left\{x \in \mathbb{C}|| x \mid \leq R_{1} \text { and } \operatorname{Im}(x)>\varepsilon\right\}\right) \text { and } V_{2}=\left\{y \in \mathbb{C}|| y \mid \leq R_{2}\right\} .
$$

Then for $R_{1}$ and $R_{2}$ large enough, and $\varepsilon$ small enough, the link $C \cap \partial\left(V_{1} \times V_{2}\right)$ in $\partial\left(V_{1} \times V_{2}\right) \simeq S^{3}$ is precisely the link $L_{C}$, and the surface $C \cap\left(V_{1} \times V_{2}\right)$ is precisely the surface $U_{C}$ in $V_{1} \times V_{2} \simeq B^{4}$. Moreover the link $C \cap \partial\left(V_{1} \times V_{2}\right)$ appears naturally as the closure of a braid $b_{C}$, that we describe in the next section.

2.2. The link $L_{C}$ as a closed braid. Recall that the group of braids with 3-strings is defined as

$$
B_{3}=\left\langle\sigma_{1}, \sigma_{2} \mid \sigma_{1} \sigma_{2} \sigma_{1}=\sigma_{2} \sigma_{1} \sigma_{2}\right\rangle .
$$

This terminology comes from the fact that there exists a natural geometric interpretation of $\sigma_{1}, \sigma_{2}$, and of the relation $\sigma_{1} \sigma_{2} \sigma_{1}=\sigma_{2} \sigma_{1} \sigma_{2}$ as depicted in Figure 6 . Note that a string of a braid is implicitly oriented from left to right. In particular if an oriented link $L$ is represented as the closure of a braid $b$, then the linking number of two sublinks $L_{1}$ and $L_{2}$ of $L$ is half the sum of all exponents of $b$ corresponding to the crossings of $L_{1}$ and $L_{2}$. 


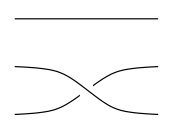

a) $\sigma_{1}$

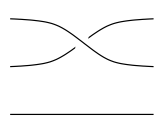

b) $\sigma_{2}$

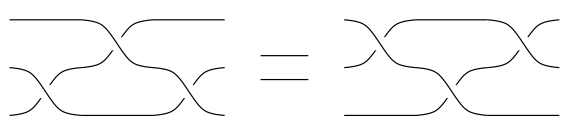

c) $\sigma_{1} \sigma_{2} \sigma_{1}=\sigma_{2} \sigma_{1} \sigma_{2}$

FigURE 6 .

Let us associate an $\mathcal{L}$-scheme to the real curve $C$. Denote by $l_{1}<l_{2}<\ldots<l_{s}$ the real vertical lines of $\mathbb{C}^{2}$ which are not transversal to $C$ (the order on the $l_{i}$ 's is the one induced by the canonical orientation of $\mathbb{R}$ ). Recall that the degree of the polynomial $Q(t)$ is $b=3 k-1$ or $b=3 k-2$.

Definition 2.7. The $\mathcal{L}$-scheme $\mathcal{L}_{C}$ realized by $C$ is given by the sequence $c_{1} c_{2} \ldots c_{s+1}$ where $c_{i}$ with $1 \leq i \leq s$ is given by

- $c_{i}=\supset_{j}$ (resp. $c_{i}=\subset_{j}$ ) if $1 \leq i \leq s$ and $l_{i}$ has an ordinary tangency point with $C$ which is a local maximum (resp. minimum) of the map $\left.\pi\right|_{\mathbb{R} C}$;

$-c_{i}=\times_{j}$ (resp. $c_{i}=\bullet_{j}$ ) if $i \leq s$ and $l_{i}$ passes through a crossing (resp. solitary node) of $\mathbb{R} C$;

(in each case $j=1$ if the transverse intersection point of $\mathbb{R} l_{i}$ and $\mathbb{R} C$ is below the second point of $\mathbb{R} l_{i} \cap \mathbb{R} C$, and $j=2$ otherwise)

and $c_{s+1}$ is given by

- $c_{s+1}=\downarrow$ (resp. $\uparrow$ ) if $b=3 k-1$, and $Q(t)$ is positive (resp. negative) for $t$ large enough;

$-c_{s+1}=\vee($ resp. $\wedge)$ if $b=3 k-2$, and $Q(t)$ is positive (resp. negative) for $t$ large enough.

Example 2.8. The $\mathcal{L}$-scheme realized by the parametrization of Example 2.5 is

$$
\bullet_{1} \subset_{1} \times_{2} \times_{1} \supset_{1} \vee \text {. }
$$

Then replace the $\mathcal{L}$-scheme $c_{1} c_{2} \ldots c_{s+1}$ by $c_{0}^{\prime} c_{1} c_{2} \ldots c_{s+1}^{\prime}$ where

- $c_{0}^{\prime}=\supset_{2}$ (resp. $\left.\supset_{1}\right)$ and $c_{s+1}^{\prime}=\subset_{1}$ if $c_{s+1}=\downarrow$ and $k$ is even (resp. odd);

- $c_{0}^{\prime}=\supset_{1}$ (resp. $\left.\supset_{2}\right)$ and $c_{s+1}^{\prime}=\subset_{2}$ if $c_{s+1}=\uparrow$ and $k$ is even (resp. odd);

- $c_{0}^{\prime}=\supset_{2}$ (resp. $\supset_{1}$ ) and $c_{s+1}^{\prime}=\subset_{1} \supset_{1} \subset_{1}$ if $c_{s+1}=\vee$ and $k$ is even (resp. odd);

- $c_{0}^{\prime}=\supset_{1}$ (resp. $\left.\supset_{2}\right)$ and $c_{s+1}^{\prime}=\subset_{2} \supset_{2} \subset_{2}$ if $c_{s+1}=\wedge$ and $k$ is even (resp. odd).

Finally replace each $\bullet_{j}$ with $\subset_{j} \supset_{j}$ and each $\times_{j}$ with $\supset_{j} \subset_{j}$ in $c_{0}^{\prime} c_{1} c_{2} \ldots c_{s+1}^{\prime}$, and do the following final substitutions:

- replace each $\supset_{j} \subset_{j}$ by $\sigma_{j}^{-1}$ (see Figure 7a);

- replace each $\supset_{1} \subset_{2}$ by $\sigma_{1}^{-1} \sigma_{2}^{-1} \sigma_{1}$ (see Figure $7 \mathrm{~b}$ );

- replace each $\supset_{2} \subset_{1}$ by $\sigma_{2}^{-1} \sigma_{1}^{-1} \sigma_{2}$ (see Figure $7 \mathrm{c}$ ).

Then we obtain a braid $b_{\mathbb{R}}$. We define the braid associated to the $\mathcal{L}$-scheme $\mathcal{L}_{C}$, denoted $b_{C}$, as the braid $b_{\mathbb{R}}\left(\sigma_{1} \sigma_{2} \sigma_{1}\right)^{k}$.

Example 2.9. After the two replacements described above, we obtain in the case of Example 2.5 


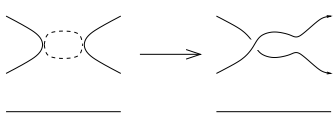

a) $\supset_{j} \subset_{j} \rightarrow \sigma_{j}^{-1}$

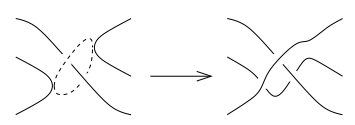

b) $\supset_{1} \subset_{2} \rightarrow \sigma_{1}^{-1} \sigma_{2}^{-1} \sigma_{1}$

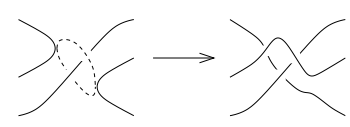

c) $\supset_{2} \subset_{1} \rightarrow \sigma_{2}^{-1} \sigma_{1}^{-1} \sigma_{2}$

FiguRE 7.

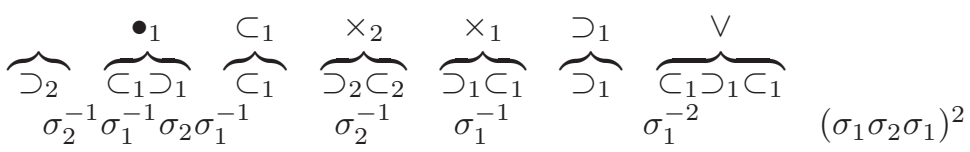

which finally gives the braid

$$
b_{C}=\sigma_{2}^{-1} \sigma_{1}^{-1} \sigma_{2} \sigma_{1}^{-1} \sigma_{2}^{-1} \sigma_{1}^{-3}\left(\sigma_{1} \sigma_{2} \sigma_{1}\right)^{2}
$$

which is the trivial braid, see Figure 8.

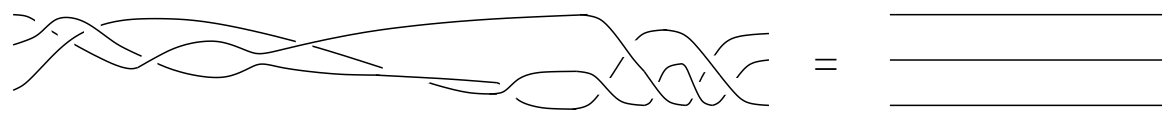

The braid $b_{C}$ of the parametrization given in Example 2.5

FiguRE 8.

Proposition 2.10. The closure of the braid $b_{C}$ is the oriented link $L_{C}$ associated to $C$.

Proof. The only part which is not already contained in [Ore99, Section 3] is the determination of the braid $C \cap \pi^{-1}(\gamma)$ where $\gamma$ is the arc in $\mathbb{C}$ parametrized by $u \in[0 ; \pi] \rightarrow \varepsilon+R_{1} \exp (2 i \pi u)$ (recall that $\varepsilon$ and $R_{1}$ have been introduced at the end of Section 2.1). But this braid is dictated by the monomials of an equation of $C$ in $\mathbb{C}^{2}$ which are dominating at infinity. Up to a real multiplicative constant, these monomials are precisely

$$
\begin{aligned}
& -y^{3}-\lambda x^{3 k-1} \text { if } c_{s+1}=\downarrow, \\
& -y^{3}+\lambda x^{3 k-1} \text { if } c_{s+1}=\uparrow, \\
& -y^{3}-\lambda x^{3 k-2} \text { if } c_{s+1}=\vee \\
& -y^{3}+\lambda x^{3 k-2} \text { if } c_{s+1}=\wedge,
\end{aligned}
$$

where $\lambda$ is some positive real number. Now the result follows from straightforward local computations as in [Ore99, Section 3].

\section{Proof of Theorem 3.9}

Here we apply the method described in the previous section to the study of the lexicographic degree of two-bridge knots. Given a long knot diagram $D$ in $\mathbb{R}^{2}$, we denote by $\bar{D}$ its projection to $\mathbb{R}^{2}$ (i.e. we forget about the sign of the crossings). 
3.1. The case of alternating diagrams. We first consider the special case of alternating diagrams, from which we will deduce the general case in Section 3.2.

Proposition 3.1. Let $D$ be the alternating trigonal diagram $C(m)$ with $m$ odd, or $C(m, n)$ with $m n$ even and positive. If $\gamma: \mathbb{R} \rightarrow \mathbb{R}^{2}$ is a polynomial map of bidegree $(3, b)$ such that $\gamma(\mathbb{R})$ is $\mathcal{L}$-isotopic to $\bar{D}$, then $b \geq\left[\frac{3 N-1}{2}\right]$, where $N$ is the crossing number of the knot.

Proof. Up to a change of coordinates in $\mathbb{C}^{2}$, we may suppose that $b=3 k-1$ or $b=3 k-2$. By genericity argument, we may also suppose that $C=\gamma(\mathbb{C})$ is a nodal curve in $\mathbb{C}^{2}$. Finally by symmetry we may further assume that $m$ is even when $m+n$ is odd.

In the case of $C(m)$, the $\mathcal{L}$-scheme of $C$ contains the patterns $\subset_{2}\left(\times_{1}\right)^{m} \supset_{2}$. In the case of $C(m, n)$, the $\mathcal{L}$-scheme of $C$ contains the patterns $\subset_{2}\left(\times_{1}\right)^{m}\left(\times_{2}\right)^{n} \supset_{1}$ (see Figure 9). By the Riemann-Hurwitz formula the map $\pi \circ \gamma$ has exactly two
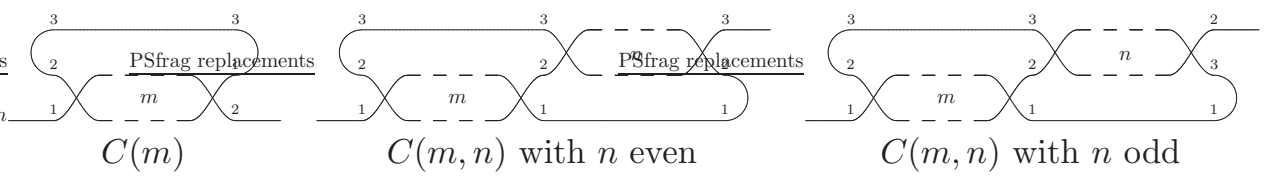

Figure 9. Diagrams for $C(m)$ or $C(m, n)$ knots

ramification points, which correspond precisely to the patterns $\subset$ and $\supset$ in the $\mathcal{L}$-scheme of $C$. In particular, these two ramification points are real. Hence, still by the Riemann-Hurwitz formula, the surface $U_{C}$ is made of three embedded disks $U_{1}, U_{2}$, and $U_{3}$. Each disk $U_{i}$ bounds the subknot $L_{i}$ of the link $L_{C}$. The positivity of self-intersections of a complex algebraic curve and Proposition 2.2 implies that $\operatorname{lk}\left(L_{i}, L_{j}\right)=U_{i} \cdot U_{j} \geq 0$. On the other hand $U_{i}$ and $U_{j}$ intersect transversally at complex conjugated nodes, thus we have

$$
\sum_{1 \leq i<j \leq 3} U_{i} \cdot U_{j}=\beta
$$

and therefore

$$
\operatorname{lk}\left(L_{i}, L_{j}\right) \leq \beta, \quad 1 \leq i<j \leq 3 .
$$

Moreover Lemma 2.4 states that

$$
N+\alpha+2 \beta=b-1 .
$$

Let us label the components $L_{1}, L_{2}$, and $L_{3}$ as depicted in Figure 9, and let us evaluate the quantity $2 \mathrm{lk}\left(L_{1}, L_{3}\right)$ from the algorithm described in Section 2.2. Clearly, no crossing point of $\mathbb{R} C$ contributes to $2 \mathrm{lk}\left(L_{1}, L_{3}\right)$, and the factor $\left(\sigma_{1} \sigma_{2} \sigma_{1}\right)^{k}$ contributes $k$ to $2 \mathrm{lk}\left(L_{1}, L_{3}\right)$. As in Section 2.2 , we denote by $c_{1} \ldots c_{s+1}$ the $\mathcal{L}$-scheme realized by $C$, and by $c_{0}^{\prime} c_{1} \ldots c_{s} c_{s+1}^{\prime}$ the result of replacing $c_{s+1}$ by $c_{0}^{\prime}$ and $c_{s+1}^{\prime}$. We have $N=n+m$ and $s=N+\alpha+2$. Let $p \geq 1$ such that $c_{p}=\subset_{2}$. Then we have $c_{p+N+1}=\supset_{2}$ and $c_{q}=\bullet_{j}$ for $1 \leq q \leq p-1$ and $N+p+2 \leq q \leq s$. We can estimate the contribution of $b_{\mathbb{R}}$ to $2 \mathrm{lk}\left(L_{1}, L_{3}\right)$ as follows: 
- in the case of $C(m)$ or $C(m, n)$ with $N$ odd:

each pattern $c_{q}$ with $p+N+1 \leq q \leq s$ contributes -1 ; each pattern $c_{q} c_{q+1}$ with $1 \leq q \leq p-1$ contributes at least -1 ; the pattern $c_{0}^{\prime} c_{1}$ contributes at least 0 ; the pattern $c_{s+1}^{\prime}$ contributes 0 if $b=3 k-1$, and -1 if $b=3 k-2$.

- in the case of $C(m, n)$ with $N$ even:

each pattern $c_{q} c_{q+1}$ with $1 \leq q \leq p-1$ or $p+N+1 \leq q \leq s-1$ contributes at least -1 , as well as the pattern $c_{0}^{\prime} c_{1}$; the pattern $c_{s} c_{s+1}^{\prime}$ contributes at least -1 if $b=3 k-1$, and at least 0 if $b=3 k-2$.

Altogether, writing $d=3 k-1-\varepsilon$, we obtain

$$
2 \operatorname{lk}\left(L_{1}, L_{3}\right) \geq k-\alpha-1-\varepsilon
$$

if $N$ is odd, and

$$
2 \operatorname{lk}\left(L_{1}, L_{3}\right) \geq k-\alpha-2+\varepsilon
$$

if $N$ is even. These last inequalities together with identities (1) and (2) give

$$
b-k \geq N-\varepsilon
$$

if $N$ is odd, and

$$
b-k \geq N-1+\varepsilon
$$

if $N$ is even. Hence we obtain

$$
b \geq\left[\frac{3 N-1}{2}\right]
$$

which proves the proposition.

Remark 3.2. The proof of Proposition 3.1 shows that if $b=\left[\frac{3 N-1}{2}\right]$ then $\beta=0$ and $\alpha=\frac{N-3}{2}$ or $\alpha=\frac{N}{2}-2$ depending on the parity of $N$. Moreover the mutual position of the solitary nodes of $C$ is imposed: the $\mathcal{L}$-scheme realized by $C$ does not contain the pattern $\left(\bullet_{j}\right)^{2}$ (i.e. the pattern corresponding to two successive solitary nodes must be $\bullet_{j} \bullet j \pm 1$ ).

Remark 3.3. Let us consider the harmonic knot $\mathrm{H}(3,7,11)$. It is proved in [KP08] that its crossing number is 6 . Then its lexicographic degree is $(3,7, c)$, which is smaller than the upper bound given in Proposition 3.1. This is the knot $6_{3}$ which is neither a torus knot nor a generalized twist knot ([KP10]). In Section 4 we shall see that the lexicographic degree of $6_{3}$ is $(3,7,11)$.

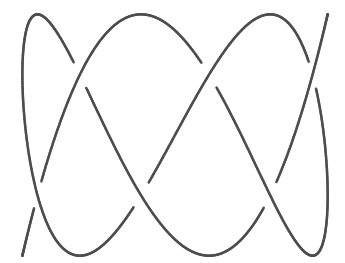

Figure 10. The harmonic knot $\mathrm{H}(3,7,11)=6_{3}$ 
3.2. Reduction to the case of alternating diagrams: real pseudoholomorphic curves. As explained in the introduction it might not be true that the lexicographic degree of a trigonal long knot diagram does not increases along an isotopy through trigonal diagrams which never increases the number of crossings. For this to be true we have to consider real pseudoholomorphic curves (see Corollary 3.7).

We consider $\mathbb{C}^{2}$ equipped with the standard Fubini-Study symplectic form

$$
\omega\left(u+i v, u^{\prime}+i v^{\prime}\right)=u \wedge v+u^{\prime} \wedge v^{\prime} .
$$

Recall that an almost complex structure $J$ on $\mathbb{C}^{2}$ is a smooth section $J: \mathbb{C}^{2} \rightarrow$ $\operatorname{End}_{\mathbb{R}}\left(T \mathbb{C}^{2}\right)$ such that $J_{p}^{2}=-I d_{T_{p} \mathbb{C}^{2}}$ for any $p \in \mathbb{C}^{2}$, and that $J$ is said to be tamed by $\omega$ if $\omega(v, J v)>0$ for any non-null vector $v \in T \mathbb{C}^{2}$. Such an almost complex structure is called real if the standard complex conjugation conj on $\mathbb{C}^{2}$ is $J$-antiholomorphic (i.e. con $j \circ J=J^{-1} \circ$ conj $)$. For example, the standard complex structure $J_{0}$ on $\mathbb{C}^{2}$ is real and tamed by $\omega$. An almost complex structure is called admissible if it is real, tamed by $\omega$, and coincide with $J_{0}$ outside a compact set in $\mathbb{C}^{2}$.

The next definition follows the lines of [OS02, Section 1] and [MS04, Chapter 2]. We also denote by $J_{0}$ (resp. conj) the standard complex structure (resp. complex conjugation) on $\mathbb{C}$.

Definition 3.4. Let $J$ be an admissible almost complex structure on $\mathbb{C}^{2}$. A real (rational) J-holomorphic curve is a map $\gamma: \mathbb{C} \rightarrow \mathbb{C}^{2}$ which is polynomial outside a compact set of $\mathbb{C}$, and such that

$$
d \gamma^{\prime} \circ J_{0}=J \circ d \gamma^{\prime} \quad \text { and } \quad \gamma \circ \operatorname{conj}=\operatorname{conj} \circ \gamma .
$$

$A$ real pseudoholomorphic curve $\gamma: \mathbb{C} \rightarrow \mathbb{C}^{2}$ is a map which is J-holomorphic for some admissible almost complex structure $J$ on $\mathbb{C}^{2}$.

Example 3.5. Every polynomial real map $\gamma: \mathbb{R} \rightarrow \mathbb{R}^{2}$ is a real $J_{0}$-holomorphic curve. However not every real pseudoholomorphic curve is $J_{0}$-holomorphic.

By definition, the image $C=\gamma(\mathbb{C})$ of any real pseudoholomorphic curve $\gamma$ coincide with a real algebraic curve $C_{0}$ outside a compact set of $\mathbb{C}^{2}$. We say that $\gamma$ is of bidegree $(3, b)$ if the curve $C_{0}$ has for Newton polygon the triangle with vertices $(0,0),(0,3)$, and $(0, b)$. As in the algebraic case, we may assume for our purposes that if a real pseudoholomorphic curve is of bidegree $(3, b)$, then $b=3 k-1$ or $b=3 k-2$. Note that nodal pseudoholomorphic curves satisfy the adjunction formula (see [MS04, Chapter 2]), in particular Lemma 2.4 still holds for pseudoholomorphic curves.

The braid theoretical approach described in Section 3 extends word by word to the case of nodal real pseudoholomorphic curves of bidegree $(3, b)$ (see for example [Ore99, OS02]). Indeed, in these latter two sections we only use the behavior at infinity of real algebraic curves and the three following facts:

(1) any intersection of two complex algebraic curves is positive;

(2) there exists a unique vertical line passing through a given point $p$ of $\mathbb{C}^{2}$, which is real if $p$ is real;

(3) the map $\pi$ is a real holomorphic map.

Let us fix an admissible almost complex structure $J$ on $\mathbb{C}^{2}$. The word by word extension of Sections 2.1 and 2.2 to $J$-holomorphic curves follows from the three following facts: 
(1') any intersection of two $J$-holomorphic curves is positive (see [MS04, Appendix E]);

$\left(2^{\prime}\right)$ there is an analogue of the pencil of vertical lines in $\mathbb{C}^{2}$ : consider the standard embedding

$$
\begin{array}{ccc}
\mathbb{C}^{2} & \longrightarrow & \mathbb{C} P^{2} \\
(x, y) & \longmapsto & {[x: y: 1]}
\end{array}
$$

then given a point $p \in \mathbb{C}^{2}$ there exists a unique $J$-holomorphic line in $\mathbb{C} P^{2}$ passing through $p$ and $[0: 1: 0]$ (see [Gro85]); by uniqueness, this line is real if $p$ is real; this produces a real pencil of "vertical" $J$-holomorphic lines on $\mathbb{C}^{2}$

(3') since $J$ is standard outside a compact set of $\mathbb{C}^{2}$, the line $\{y=b\}$ is $J$ holomorphic for $|b|$ large enough; replace the map $\pi$ by the map $\pi_{b}: \mathbb{C}^{2} \rightarrow$ $\{y=b\}$ which associates to each point $p$ of $\mathbb{C}^{2}$ the (unique) intersection point of $\{y=b\}$ with the $J$-holomorphic vertical line passing through $p$; the map $\pi_{b}$ is clearly a real $J$-holomorphic map.

The main advantage in dealing with real pseudoholomorphic curves rather than real algebraic curves is that we have the following proposition.

Proposition 3.6 (Orevkov, [Ore99]). Let $\gamma: \mathbb{C} \rightarrow \mathbb{C}^{2}$ be a real nodal pseudoholomorphic curve of bidegree $(3, b)$ realizing an $\mathcal{L}$-scheme $\mathcal{L}_{\gamma}$, and let $\mathcal{L}_{\gamma}^{\prime}$ be the $\mathcal{L}$-scheme obtained from $\mathcal{L}_{\gamma}$ by one of the following elementary operations :

$$
\begin{gathered}
\times_{j} \supset_{j \pm 1} \leftrightarrow \times_{j \pm 1} \supset_{j} \quad \subset_{j \pm 1} \times_{j} \leftrightarrow \subset_{j} \times_{j \pm 1} \quad \times_{j} \times_{j} \rightarrow \emptyset \\
\times_{j} \supset_{j} \leftrightarrow \supset_{j} \bullet_{j} \quad \subset_{j} \times_{j} \leftrightarrow \bullet_{j} \subset_{j} \quad \times_{j+1} \times_{j} \times_{j+1} \leftrightarrow \times_{j} \times_{j+1} \times_{j}
\end{gathered}
$$

Then there exists a real nodal pseudoholomorphic curve of bidegree $(3, b)$ realizing $\mathcal{L}_{\gamma}^{\prime}$.

Corollary 3.7. Let $D_{1}$ and $D_{2}$ be two trigonal long knot diagrams such that $D_{2}$ is obtained from $D_{1}$ by an isotopy of trigonal diagrams which never increase the number of crossings. If there exists a real nodal pseudoholomorphic curve $\gamma_{1}: \mathbb{C} \rightarrow$ $\mathbb{C}^{2}$ of bidegree $(3, b)$ such that $\gamma_{1}(\mathbb{R})$ is $\mathcal{L}$-isotopic to $\bar{D}_{1}$, then there also exists a real nodal pseudoholomorphic curve $\gamma_{2}: \mathbb{C} \rightarrow \mathbb{C}^{2}$ of bidegree $(3, b)$ such that $\gamma_{2}(\mathbb{R})$ is $\mathcal{L}$-isotopic to $\bar{D}_{2}$.

Proposition 3.8. Let $K$ be the two-bridge knot $C(m)$ with $m$ odd, or $C(m, n)$ with $m n$ positive and even, and let $N$ be its crossing number. Let $D$ be any trigonal diagram of $K$. If there exists a real nodal pseudoholomorphic curve $\gamma: \mathbb{C} \rightarrow \mathbb{C}^{2}$ of bidegree $(3, b)$ such that $\gamma(\mathbb{R})$ is $\mathcal{L}$-isotopic to $\bar{D}$, then $b \geq\left[\frac{3 N-1}{2}\right]$.

Proof. According to Corollary 3.7 and Theorem A, it is enough to prove the proposition when $D$ is alternating, which has been done in Proposition 3.1 (recall that real pseudoholomorphic curves satisfy Lemma 2.4, so the proof of Proposition 3.1 extends word by word to this case).

Theorem 3.9. Let $K$ be the two-bridge knot $C(m)$ with $m$ odd, or $C(m, n)$ with $m n$ positive and even, and let $N$ be its crossing number. Then the lexicographic degree of $K$ is

$$
\left(3,\left[\frac{3 N-1}{2}\right],\left[\frac{3 N}{2}\right]+1\right) .
$$


Proof. Let $K$ be any two-bridge knot. It is proved in [KP10] that there exists a polynomial curve $\gamma: \mathbb{R} \rightarrow \mathbb{R}^{3}$ of degree $(3, b, c)$ such that $N<b<c, b+c=3 N$, and $\gamma(\mathbb{R})$ is the knot $K$. In particular we have $b \leq\left[\frac{3 N-1}{2}\right]$ and $c \geq\left[\frac{3 N}{2}\right]+1$. If $K$ is $C(m)$ or $C(m, n)$, then by Proposition 3.8 we have $b \geq\left[\frac{3 N-1}{2}\right]$. This implies that $c \leq\left[\frac{3 N}{2}\right]+1$, and the theorem is proved.

Remark 3.10. It is shown in [KP11] that the harmonic knot $\mathrm{H}(3,3 n+2,3 n+1)$ is the torus knot $\mathrm{T}(2,2 n+1)=C(2 n+1)$. By Theorem 3.9, this is an explicit polynomial parametrization of minimal lexicographic degree $(3,3 n+1,3 n+2)$. In this example, the number of crossings of the diagram is greater than the crossing number of the knot. In [KP09] it is proved that there exists a polynomial representation of $\mathrm{T}(2,2 n+1)$ which is of minimal degree, and such that the number of crossings is minimal.

\section{A LOWER BOUND}

Theorem 4.3 provides a general lower bound for the lexicographic degree of every non-trivial knot, furthermore this lower bound is sharp.

Proposition 4.1. Let $\gamma: \mathbb{R} \rightarrow \mathbb{R}^{3}$ be a polynomial map of degree $(a, b, c)$ whose image is a smooth knot. Suppose that $a$ and $b$ are coprime, and that the xy-diagram of $\gamma(t)$ is alternating with $(a-1)(b-1) / 2$ crossings. Then $c \geq a b-a-b$.

The proof of Proposition 4.1 uses the following lemma of Frobenius type.

Lemma 4.2. Let $a$ and $b$ be coprime positive integers. The number of integers of the form $n=\alpha a+\beta b, n \leq a b-a-b-1,(\alpha, \beta) \in \mathbb{Z}_{\geq 0}^{2}$, is equal to $(a-1)(b-1) / 2$.

Proof. Let $N=(a-1)(b-1) / 2$. First, let us show that the integer $2 N-1=a b-a-b$ is not of the form $\alpha a+\beta b$, with $(\alpha, \beta) \in \mathbb{Z}_{\geq 0}^{2}$.

If we had $a b-a-b=\alpha a+\beta b$, then $\alpha \equiv-1(\bmod b)$, and $\alpha \geq b-1$. Hence we deduce that $\alpha a+\beta b \geq a b-a+\beta b>2 N-1$, contrary to our hypothesis

Now consider the sets $E=\left\{(\alpha, \beta) \in \mathbb{Z}_{\geq 0}^{2}, \alpha a+\beta b \leq 2 N-2\right\}$ and $Q=\{(\alpha, \beta) \in$ $\left.\mathbb{Z}_{>0}^{2}, \alpha \leq b-2, \beta \leq a-2\right\}$. The set $Q$ has $2 N$ elements, and contains $E$. Denoting $s(\alpha, \beta)=\alpha a+\beta b, \alpha^{\prime}=b-2-\alpha$, and $\beta^{\prime}=a-2-\beta$, we have $s(\alpha, \beta)+s\left(\alpha^{\prime}, \beta^{\prime}\right)=$ $2(2 N-1)$, and then $\left(\alpha^{\prime}, \beta^{\prime}\right) \in Q-E$ if and only if $(\alpha, \beta) \in E$.

Consequently, half the elements of $Q$ are in $E$, and then $E$ has $N$ elements. Since $a$ and $b$ are coprime, the mapping $s$ is an injection from $E$ to $\mathbb{Z}_{\geq 0}$, which concludes the proof.

Proof of Proposition 4.1. The $N=(a-1)(b-1) / 2$ crossing points of the $x y$ projection of $\gamma(t)=(x(t), y(t), z(t))$ correspond to parameter pairs $\left(s_{i}, t_{i}\right)$ such that

$$
x\left(t_{i}\right)=x\left(s_{i}\right), \quad y\left(t_{i}\right)=y\left(s_{i}\right), \quad z\left(t_{i}\right)>z\left(s_{i}\right) .
$$

Consider $V$ the vector subspace of $\mathbb{R}[t]$ generated by the polynomials $x(t)^{\alpha} y(t)^{\beta}$, $\alpha a+\beta b \leq 2 N-2$. By Lemma 4.2, the dimension of $V$ is $N$. Let us define the linear mapping $\varphi: V \rightarrow \mathbb{R}^{N}$ by $\varphi(h)=\left(h\left(t_{1}\right), \ldots, h\left(t_{N}\right)\right)$. If a polynomial $h$ is in the kernel of $\varphi$, then $h\left(t_{1}\right)=h\left(s_{1}\right)=\cdots=h\left(t_{N}\right)=h\left(s_{N}\right)=0$, and the polynomial $h$ has $2 N$ distinct roots. Since $\operatorname{deg}(h) \leq 2 N-2$, we deduce that $h=0$. 
Consequently the mapping $\varphi$ is an isomorphism and there exists $h \in V$ such that $h\left(t_{i}\right)=h\left(s_{i}\right)=\left(z\left(t_{i}\right)+z\left(s_{i}\right)\right) / 2$.

Let us define $\tilde{z}(t)=z(t)-h(t)$. The images of $\gamma$ and $\tilde{\gamma}(t)=(x(t), y(t), \tilde{z}(t))$ are isotopic and realize the same diagram. By construction, at each crossing we have $\tilde{z}\left(t_{i}\right)>0>\tilde{z}\left(s_{i}\right)$.

Let us write $\left\{t_{1}, \ldots, t_{N}, s_{1}, \ldots, s_{N}\right\}=\left\{\tau_{1}, \ldots, \tau_{2 N}\right\}$ where $\tau_{j}<\tau_{j+1}$. Since the diagram realized by $\tilde{\gamma}(t)$ is alternating, we may assume that $(-1)^{i} \tilde{z}\left(\tau_{i}\right)>0$. Consequently the polynomial $\tilde{z}(t)$ has at least one root in the interval $\left(\tau_{j}, \tau_{j+1}\right)$, and $\operatorname{deg}(\tilde{z}) \geq 2 N-1$. Since $\operatorname{deg}(h) \leq 2 N-2$, we conclude that $\operatorname{deg}(z) \geq 2 N-1$.

As a consequence we deduce:

Theorem 4.3. Let $K$ be a knot of crossing number $N \neq 0$. Then the lexicographic degree of $K$ is at least $(3, N+1,2 N-1)$. For every integer $N \not \equiv 2(\bmod 3), N \geq 3$, there exists a knot of crossing number $N$ and lexicographic degree $(3, N+1,2 N-1)$

Proof. Let $(a, b, c)$ be the lexicographic degree of $K$. Since $K$ is nontrivial, we have $a \geq 3$. Suppose that $a=3$. Then $K$ is a two-bridge knot, and $b$ is not divisible by 3. Moreover by Bézout's theorem we have $N \leq(a-1)(b-1) / 2=b-1$, that is $b \geq N+1$.

Let $\gamma(t): \mathbb{R} \rightarrow \mathbb{R}^{3}$ be a polynomial parametrization of $K$ of degree $(3, b, c)$. If $b=N+1$, then the $x y$-diagram of $\gamma(t)$ has the minimal number of crossings. Since $K$ is a two-bridge knot, it is an alternating knot, and the $x y$-diagram of $\gamma(t)$ is alternating. By Proposition 4.1, we conclude that $c \geq 2 N-1$.

On the other hand, when $N \not \equiv 2(\bmod 3)$, the harmonic knot $\mathrm{H}(3, N+1,2 N-1)$ has crossing number $N$ (see [KP08]), hence its lexicographic degree is $(3, N+1,2 N-$ $1)$.

We also obtain the lexicographic degree of an infinite family of three-bridge knots.

Corollary 4.4. Let $\mathrm{H}$ be the harmonic knot $\mathrm{H}=\mathrm{H}(5, b, 4 b-5)$, with $b \not \equiv 0(\bmod 5)$. The knot $\mathrm{H}$ is a three-bridge knot of lexicographic degree $(5, b, 4 b-5)$.

Proof. The $x y$-projection of the knot $\mathrm{H}$ has $N=2(b-1)$ crossings and is alternating (see [KP11]). If $\mathrm{H}$ was two-bridged, then it would have a minimal diagram which would be alternating and two-bridged. By Tait's flyping conjecture, this is not the case since the $x y$-projection of $\mathrm{H}$ is alternating and three-bridged.

Let $(x(t), y(t), z(t))$ be a polynomial knot isotopic to H. Since $\mathrm{H}$ is not two-bridged, we have $\operatorname{deg}(x) \geq 5$. If $\operatorname{deg}(x)=5$, then by Bézout's theorem we have $N \leq$ $2(\operatorname{deg}(y)-1)$, that is $\operatorname{deg}(y) \geq b$. If $\operatorname{deg}(y)=b$, then by Proposition 4.1 we obtain $\operatorname{deg}(z) \geq 5 b-5-b=4 b-5$.

Remark 4.5. The lexicographic degree of the harmonic knot $\mathrm{H}(a, b, a b-a-b)$ is not always $(a, b, a b-a-b)$. For example, if $a=4$ this knot is two-bridged and has a polynomial parametrization of degree $\left(3, b^{\prime}, c^{\prime}\right)$, which is lexicographically smaller than $(4, b, 3 b-4)$.

Proposition 4.1 can also be used to study the maximal degree of a polynomial knot, that is the maximum degree of its components. 
Corollary 4.6. Let $K$ be a polynomial knot of degree $d$ and crossing number $N$. We have

$$
N \leq \frac{(d-2)(d-3)}{2}
$$

Moreover, if $d>5$ and if $K$ is alternating, then we have:

$$
N \leq \frac{(d-1)(d-4)}{2} .
$$

Proof. Using an affine transformation, we can suppose that the lexicographic degree of $K$ is $\leq(d-2, d-1, d)$. By Bézout's theorem we see that the number of crossings of the $x y$-projection is at most $(d-2)(d-3) / 2$, which proves the first assertion. If we had $N=(d-2)(d-3) / 2$, then the lexicographic degree of $K$ would be $(d-2, d-1, d)$. By Proposition 4.1, we have $d \geq(d-2)(d-1)-(d-2)-(d-1)=d^{2}-5 d+5$, which is impossible, since $d>5$. Consequently, we have $N \leq(d-2)(d-3) / 2-1=$ $(d-1)(d-4) / 2$.

Remark 4.7. Since knots of crossing number smaller than 8 are alternating, we deduce that the only knot of degree 4 is the trivial knot, the only nontrivial knot of degree 5 is the trefoil, and the crossing number of a knot of degree 6 is at most 5.

\section{REFERENCES}

[BKP14] E. Brugallé, P. -V. Koseleff, D. Pecker. Untangling trigonal diagrams, 2014

[Cr04] P. R. Cromwell, Knots and links, Cambridge University Press, Cambridge, 2004. xviii+328 pp.

[Gro85] M. Gromov. Pseudoholomorphic curves in symplectic manifolds. Invent. Math., 82(2):307-347, 1985.

[KP08] P. -V. Koseleff, D. Pecker, On polynomial Torus Knots, Journal of Knot Theory and its Ramifications, Vol. 17 (12) (2008), 1525-1537

[KP09] P. -V. Koseleff, D. Pecker, A polynomial parametrization of torus knots. Appl. Algebra Engrg. Comm. Comput. Vol 20 (5-6) (2009), 361-377

[KP11] P. -V. Koseleff, D. Pecker, Chebyshev knots, Journal of Knot Theory and Its Ramifications, Vol 20, 4 (2011) 1-19

[KP10] P. -V. Koseleff, D. Pecker, Chebyshev diagrams for two-bridge knots, Geom. Dedicata 150 (2010), 405-425

[KP12] P. -V. Koseleff, D. Pecker, Harmonic knots, 18p., arXiv1203.4376

[MS04] D. McDuff, and D. Salamon. J-holomorphic curves and symplectic topology Am. Math. Soc. Coll. Publi. volume 52, 2004

[Mi00] R. Mishra, Minimal degree sequence for torus knots, Journal of Knot Theory and its Ramifications, Vol. 9 (2000), no 6, 759-769.

[Ore99] S. Yu. Orevkov. Link theory and oval arrangements of real algebraic curves. Topology, 38(4):779-810, 1999.

[OS02] S. Yu. Orevkov and E. I. Shustin. Flexible, algebraically unrealizable curves: rehabilitation of Hilbert-Rohn-Gudkov approach. J. Reine Angew. Math., 551:145-172, 2002.

[RS96] A. Ranjan and R. Shukla, On polynomial representation of torus knots, Journal of knot theory and its ramifications, Vol. 5 (2) (1996) 279-294.

[Sh92] A.R. Shastri, Polynomial representation of knots, Tôhoku Math. J. 44 (1992), 11-17.

[Va90] V. A. Vassiliev, Cohomology of knot spaces, Theory of singularities and its Applications, Advances Soviet Maths Vol 1 (1990)

Erwan BRUGallé

École Polytechnique, Centre Mathématiques Laurent Schwartz, 91128 Palaiseau Cedex, France

e-mail: erwan.brugalle@math.cnrs.fr 
Pierre-Vincent KoselefF

Université Pierre et Marie Curie (UPMC Sorbonne Universités),

Institut de Mathématiques de Jussieu (IMJ-PRG) \& Inria-Rocquencourt

e-mail: koseleff@math.jussieu.fr

Daniel PECKER

Université Pierre et Marie Curie (UPMC Sorbonne Universités),

Institut de Mathématiques de Jussieu (IMJ-PRG),

e-mail: pecker@math.jussieu.fr 